\title{
How to follow metabolic clues to find cancer's Achilles heel
}

\author{
Labs combine techniques to study why cancer cells are so metabolically flexible.
}

\author{
Vivien Marx
}

$\mathrm{S}$ uffocation seems an apt way to kill a tumor. But merely cutting off the oxygen supply doesn't do the trick. "Interfering with one of the two reactions furnishing energy to tumor cells is not enough to kill them," noted Otto Warburg and colleagues from the Kaiser Wilhelm Institute for Biology in $1927^{1}$. "It is necessary to stop both respiration and fermentation, if the cells are to be killed for want of energy."

Tumor cells are sugar-holics. This high sugar consumption is, as Warburg and others found, an addiction that fuels these cells' high proliferation rate. In healthy eukaryotic tissue, cells metabolize glucose to pyruvate, then to lactate or, through the steps of mitochondrial respiration that involve oxygen consumption, to $\mathrm{CO}_{2}$. Cancer cells can shift how they generate energy: they usually 'choose' the conversion of glucose to pyruvate and avoid mitochondrial oxidation, and do so both when oxygen is present and when it is absent. This metabolic particularity is called the Warburg effect. It's well known but not completely deciphered, so researchers are trying new, multimodal approaches.

At first glance it seems odd that cancer cells choose a metabolically less efficient mode of adenosine $5^{\prime}$-triphosphate (ATP) production. As researchers Matthew Vander Heiden, Lewis Cantley and Craig Thompson point out, metabolizing one molecule of glucose to lactate without oxygen leads to two molecules of ATP, whereas oxidative phosphorylation, in the sequence of chemical reactions in eukaryotic mitochondria called the tricarboxylic acid cycle (TCA), can convert one glucose molecule into 36 ATPs $^{2}$. Teasing out the links between metabolism and cancer "remains an exciting area of investigation," they note. The cancer cell's choice is less efficient, but it's faster and helps the cells speedily assemble building blocks for proteins, lipids, nucleotides and other macromolecules. Glucose also propels signaling pathways: the phosphoinositide 3-kinase pathway is involved in growth and proliferation and is frequently dysregulated in cancer.

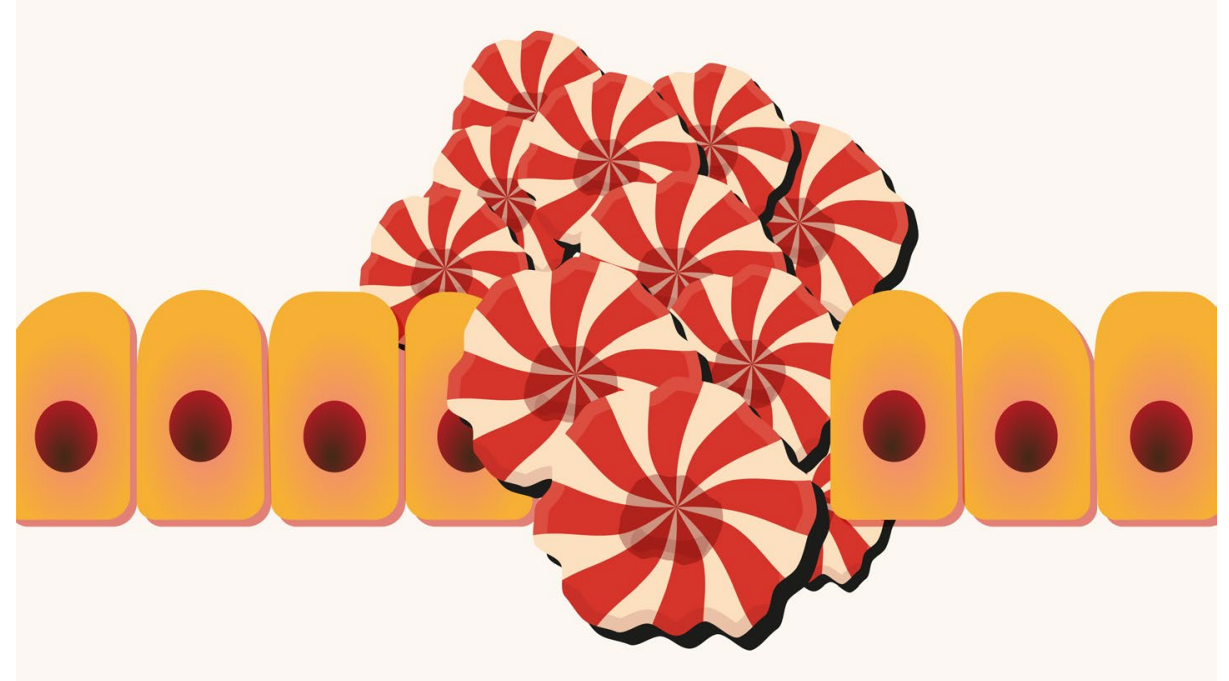

Tumor cells are sugar-holics. Their sugar consumption fuels their high proliferation rate. Credit: E. Dewalt and J. Carmona Sanchez/Springer Nature

\section{Metabolite profiling}

When researchers Thompson, Ralph De Berardinis and colleagues characterized the metabolism of glioblastoma cells with ${ }^{13} \mathrm{C}$ nuclear magnetic resonance (NMR) spectroscopy, they confirmed that glucose metabolism in those cells mainly takes place through aerobic glycolysis, even when cells have ample oxygen at their disposal ${ }^{3}$. And they found that high glutamine metabolism of cancer cells feeds the production of proteins and nucleotides and supports biosynthetic pathways such as fatty acid synthesis. Characterizing cancer's glutamine addiction is a way to explore tumor metabolism and paths to hinder tumor growth, says Daniel Raftery, who splits his time between his metabolomics lab and a core facility at the University of Washington and the Fred Hutchinson Cancer Research Center. He and his team looked at how the glutaminase inhibitor bis-2-(5-phenylacetamido-1,3,4-thiadiazol2-yl)ethyl sulfide (BPTES) metabolically affects different breast cancer cell lines ${ }^{4}$. The oncogene MYC in human breast cancer cells increases production of the enzyme glutaminase, which is involved in glutamine metabolism. Using NMR spectroscopy and isotope tagging with ${ }^{15} \mathrm{~N}$-cholamine, they identified 41 metabolites, many of which are involved in glucose and glutamine metabolism, as well as in amino acid synthesis. They found marked metabolite changes in two breast cancer cell lines that revealed their BPTES vulnerability. The experiments were performed at both normal and hypoxic oxygen levels. Cancer cells in vivo are often oxygen starved, which might promote the metabolic shift away from mitochondrial respiration toward routes enabling more aggressive growth.

Profiling metabolites is a way to assess metabolic changes in a given cancer and, potentially, disease development or treatment success, says Raftery. Methods have progressed, and metabolomics now resides alongside other 'omics technologies: genomics, proteomics, transcriptomics. By helping to track dynamic changes in biological systems, metabolomics complements other 'omics. "The hope is that 


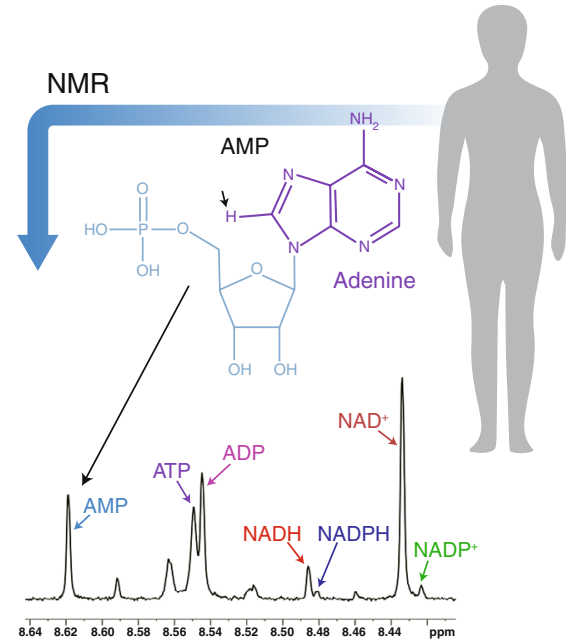

The Raftery lab can measure seven metabolic coenzymes at once. The $\mathrm{H}$ atom (arrow) in the coenzymes' adenine component is the detected NMR 'fingerprint'. Adapted from ref. ${ }^{5}$ with permission from ACS. Credit: D. Raftery, U. Washington; E. Dewalt/Springer Nature

metabolomics is going to explain a lot more," he says, in that a biochemical snapshot of a cell or tissue integrates genomic and proteomic effects. Mass-spectrometry-based analysis of serum can tease out metabolic cancer signatures. Raftery and his team used targeted liquid chromatography-tandem mass spectrometry to compare samples from people with colon cancer, people with colon polyps and those with healthy colons. The scientists looked at 20 chemical classes of metabolites in 25 pathways, detected 113 of the 158 targeted metabolites and found distinct signatures for each group of people.

Mass spectrometry has advanced more quickly in recent years than NMR, the mass spec community is larger, the technique is more sensitive than NMR and it can detect many more metabolites, he says. He likes NMR's precision for quantifying metabolites. In tracer studies, NMR helps with following specifically labeled glucose through pathways. "NMR will tell you which atom is labeled," he says. Separately, he and his team developed an NMR-based method to measure seven metabolic coenzymes at once $^{5}$. To do so, among other tasks, the team developed a library of NMR chemical shift data for identifying these metabolites.

At his core facility, Raftery and colleagues help labs identify as many metabolites as possible. Untargeted metabolomics can deliver numerous unknowns, he says. Many detected metabolites can be "rabbit holes": it's hard to assess their biological roles. "You usually end up with sort of a hairball of correlations," he says. To avoid rabbit holes and hairballs, he and his team have developed targeted assays: a list of 300 aqueous metabolites from 55 pathways. It's not full coverage, he says, but it covers important aspects of the TCA and the pentose phosphate pathway, which runs parallel to glycolysis, amino acid metabolism and nucleotide synthesis.

With cancer, important metabolic changes tend to be quite pronounced, says Raftery. In brain cancer, for example, mutation of enzymes such as isocitrate dehydrogenase (IDH-1) is common and there are readily identifiable metabolites such as 2-hydroxyglutarate. Yet glycolysis can change for many reasons, warranting metabolomic analyses. It's also challenging to study subtle metabolic changes in cancer cells. And although it's measurable and perhaps promising in some cancers, achieving partial metabolic inhibition with a potential cancer drug is also hard, says Raftery.

Metabolomics wrestles with quality issues, sometimes due to a lack of standardized sample prep methods, says Raftery. The field's newcomers are less familiar with protocols, and they might not run controls or assure that instruments run well. Quality issues are being addressed by the National Cancer Institute's recently assembled Metabolomics Quality Assurance and Quality Control Consortium, of which Raftery is a member, along with others in academia and industry. NMR data are, in his experience, more readily reproducible than mass spec data. "In terms of global profiling, the data quality is still problematic, I would say," but targeted profiling has been improving lab data quality.

Beyond developing best practices for the metabolomics community, the consortium is working on a set of reference samples for testing instruments, mainly for global liquid chromatography-mass spec (LC-MS)-based work where there are reproducibility issues, says Raftery. NIST SRM 1950 is the existing reference material for blood, but none exists for urine. Combined reference materials could help, such as one with 300 metabolites. If it runs well, "then you are probably going to get the right identification in your real samples," he says.

\section{Live cell assessment}

To assess the metabolic state of live cells, some labs might use the XF Analyzer, an instrument that measures mitochondrial respiration and glycolysis in multiwell plates, developed by Seahorse Bioscience, which was acquired by Agilent Technologies. "Metabolism is by far the best way to both measure and explain biology, because it's the working end of biology," says David Ferrick, a researcher who develops market strategy for Agilent's cell analysis division and is Seahorse's former chief scientific officer. Cancer genes, pathways and signaling set up the work, he says; "what does the work is the metabolic system." When Ferrick joined Seahorse, he advanced Analyzer development for metabolism labs. At the time, many researchers sought to assess mitochondrial metabolism in the context of aging, neurodegeneration and diabetes. Cancer researchers such as Thompson and others were "reinventing Warburg," he says, but cancer cell metabolism research was still gaining momentum. The XF Analyzer uses two types of fluorophores as biosensors: one binds oxygen, which quenches fluorescence to capture the output of mitochondrial respiration; another binds protons to capture glycolysis. Although the fluorophores were well known, they were hard to apply. "Try to adhere something that hates water to a piece of plastic and then put it in water, which is essentially media," says Ferrick. A readout is generated when cartridges equipped with these fluorophores are lowered into multiwell plates to within 200 micrometers of the cell monolayer.

At Agilent, Ferrick's colleague Steven Fischer is part of the NCI metabolomics quality consortium, and the two men regularly discuss metabolism research. Metabolomics analysis can help with exploring the details of shutting down malignant cancer cell growth. In Ferrick's view, "molecular metabolomics is probably the best way to know that you've got it right." But using metabolomics to more globally compare cancer cells and healthy cells-“that doesn't always work out," he says. As sequencing and 'omics have advanced, labs have discovered many genes of unknown function they must assay, says Ferrick. Instead of this "forward genetics" approach, he hopes labs can return to "reverse genetics": start with the biology, such as a metabolic change, explore this change's molecular details, pursue its metabolic underpinnings.

A single modality cannot "tell you the whole story," says Ferrick. The same metabolites in a tumor might be involved in different activity in a different tumor type, which means a functional readout needs

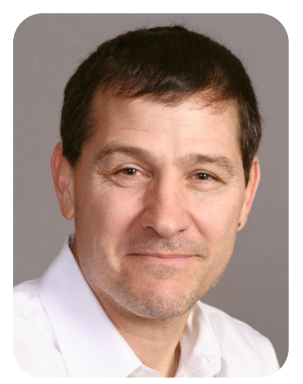

As "the working end of biology," metabolism is best for measuring and explaining biology, says David Ferrick.

Credit: Agilent Technologies 
to be combined with molecular details. He recommends that cancer metabolism researchers "don't go too far in one modality or the other. Iterate." After a molecular assay, labs might explore function, or after a metabolomic assay they might do a cell biology experiment. Sequencing is powerful, and yet it has meant "the cart got in front of the horse." He hopes labs can use "cellcentric" approaches for integrating genomic, proteomic and metabolic findings.

Cell-centricity is an opportunity for vendors, he says, to help labs not just focus on their "favorite assay" but obtain the molecular, metabolic and functional information needed in cancer research. Cancer cells are metabolically flexible: they metabolize nutrients and grow rapidly, and their microenvironment changes, which in turn affects their pathology. They adapt and maintain homeostasis throughout. "Cancer is in a phenomenal equilibrium," and a question that intrigues him is, "what keeps it in equilibrium?"

\section{The right food}

"In tissue culture, any cell that grows rapidly is a sugar-holic," says Lydia Finley, a cell biologist and metabolism researcher at Memorial Sloan Kettering Cancer Center. Cancer research labs have traditionally grown cells in media rich in glucose and glutamine, but it's unclear, she says, whether that is what cells want in culture. Cells "always amaze me by how beautifully they solve the problem of how to meet their metabolic needs," she says. "They're not dumb, so if you give them a lot of glucose, they use it." In vivo, cancer cells are often in physiologic starvation mode, which has made it challenging for metabolism researchers to decide how best to model the "cancer cell experience."

Cancer cells cultured in static media eventually experience glucose starvation, which is not physiological, says Rockefeller University researcher Kivanç Birsoy. As a postdoctoral fellow in the lab of David Sabatini at the Whitehead Institute for Biomedical Research, Birsoy and postdoc bioengineer Richard Possemato, who is now on the NYU faculty, developed Nutrostat ${ }^{6}$. In this continuous-flow device, cancer cells are cultured in an environment more like their typical low-glucose habitat. The device flows new media in continuously and removes waste. Birsoy assessed commercially available cell culture media such as Roswell Park Memorial Institute (RPMI) medium and Dulbecco's modified Eagle's medium (DMEM) and realized they were geared for growing cells and not right for studying "real" cancer cell metabolism. They feed the cells too well with oxygen, nutrients and lipids.

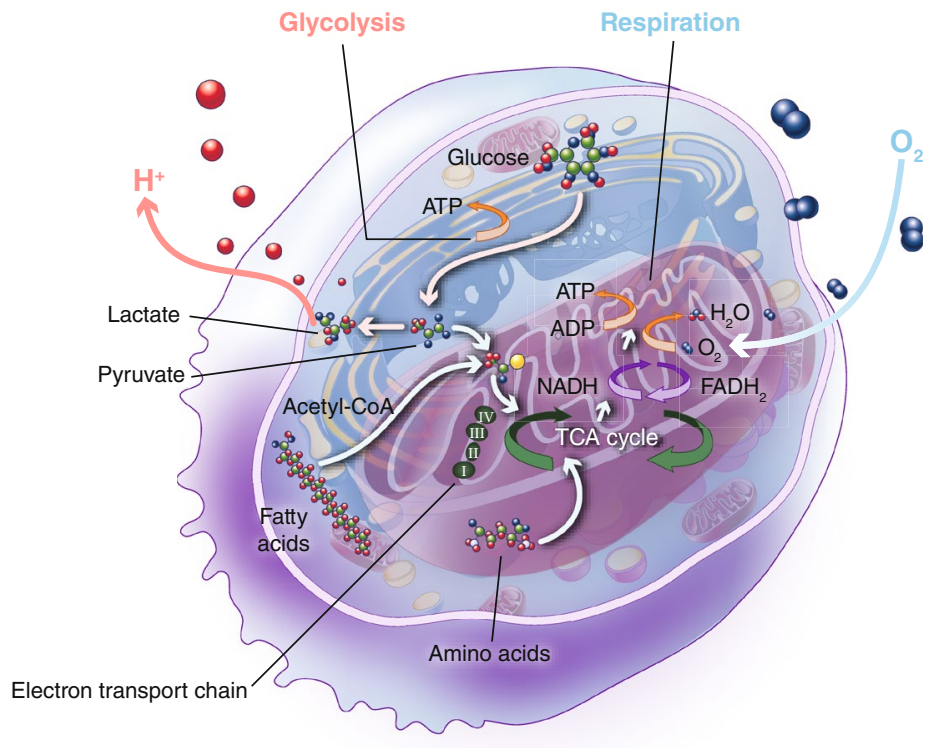

Cancer cells are shifty. For energy generation, they usually 'choose' conversion of glucose to pyruvate and avoid mitochondrial oxidation, whether oxygen is present or absent. Credit: Agilent Technologies; E. Dewalt/Springer Nature

"Cancer cells in real tumors, they are exposed to much less nutrients, they're starved for oxygen, they're starved for glucose." A wider variety of media formulations is only beginning to emerge, he says.

\section{Going multimodal}

Birsoy's sense is that the Whitehead Sabatini lab was one of the first to combine mammalian genetics, CRISPR-based genetic screens and the study of cellular metabolism. Birsoy has situated his cancerfocused Rockefeller University lab at this interface. "We come from genetics, so we do metabolomics based on the things we get from genetics," he says. The work involves human cells and mice, biochemical assays and targeted mass spectrometry. "We do a lot of CRISPR screens and we do a lot of biochemistry," he says. Yeast research has long leveraged genetics to study metabolism, but tools were lacking for mammalian cells. CRISPR-Cas9-based screens have enabled the melding of genetics and metabolism. He and others used shRNA screens and battled offtarget effects. CRISPR screens deliver more robust results. Tool development has taken a while also because metabolism research, highly active in the 1970s and 1980s, began to re-emerge only at the turn of the 21st century.

While in the Sabatini lab, Birsoy used Nutrostat to study the metabolism of different types of patient-derived cancer cells in low-glucose conditions ${ }^{6}$. He and his colleagues did a genetic screen and applied lentiviral DNA barcodes to label and track cells. The cells switched to mitochondrial oxidative phosphorylation (OXPHOS). The team detected which mitochondrial mutations affected cancer cells the most, which revealed cancer cell types especially vulnerable to OXPHOS inhibitors. Using DNA barcoding and Nutrostat, he and his team more recently looked at cancer cells that, unlike most cancer cells, cannot make their own cholesterol ${ }^{7}$. In the rare and aggressive anaplastic large-cell lymphoma, cells draw cholesterol from lipoproteins in their environment. Using CRISPR-Cas9based screens, cell barcoding and metabolite profiling, Birsoy's team has found this cancer cell's liability: it's the LDL receptor, which is part of the cholesterol-uptake pathway. They also looked at the pathway's biochemical intermediates. The cells have lost expression of an enzyme in the middle of that pathway, and the substrate for that enzyme, squalene, accumulates massively in these cells.

The loss of cholesterol-synthesis ability struck Birsoy as curious. It appears squalene has antioxidant properties, which turns this loss into a cellular survival advantage. This research is part of Birsoy's larger quest to explore the metabolic heterogeneity of tumors across cancer types and within tumors. Potentially, cancers can be discerned according to metabolic traits. "That's my goal," he says. Rare cancer types, such as the lymphoma he most recently studied, reveal specific metabolic vulnerabilities, he says. "I don't think there is a common metabolic vulnerability that is universal to all cancers." 
Birsoy is now applying Nutrostat to characterize cancer cells' use of other nutrients, such as glutamine. That amino acid is important in protein synthesis and feeds the TCA cycle in ways that render it almost as metabolically important as glucose, he says. One misconception that haunts cancer research, he says, is that cancer cells use a lot of glucose because of mitochondrial damage. The opposite might be true: they might be more dependent on their mitochondria and switch to OXPHOS as needed. "They use their mitochondria to survive," he says.

For experiments, he has used XF Analyzers and finds the instruments helpful for exploring perturbations. Those findings need follow-up with metabolic profiling and tracer experiments to track where metabolites go. Birsoy hopes new types of biosensors will be developed that give functional and quantitative readouts of cellular metabolites. "It's a very underdeveloped field," he says. As with other 'omics techniques such as transcriptomics, mass spec can deliver too much data, he says. His line of inquiry starts with a focus on a particular metabolic aspect or pathway and not with a list of all of a cell's possible metabolites. "That's why I like genetics," he says. "We go after that pathway using metabolomic tools, then it's usually successful," he says, both with cancer cells and healthy cells.

\section{Stem cells, cancer cells}

One half of Finley's metabolism-focused lab at MSKCC is devoted to stem cells, and the other to cancer cells. Metabolically, as proliferative cells, they have much in common, she says ${ }^{8}$. Fewer scientific studies focus on stem cell metabolism than on cancer cell metabolism, but she speculates that the functional and metabolic plasticities of stem cells are connected.

When studying metabolism, labs might be tempted to measure all the metabolites of a given cell, but "it almost always is inherently meaningless in and of itself," she says. These metabolites are good to know when a lab knocks out an enzyme in, for example, the glycolysis pathway and wants to study how and where the pathway is perturbed. But in a comparison of a KRASmutant and KRAS-wild-type cell, or when NMR or mass spec delivers data about cancer cell metabolites after treatment with a receptor tyrosine kinase inhibitor, the metabolites in and of themselves don't offer the picture she seeks.

Members of Finley's lab can get uneasy when a metabolite does not change the same way it did in their last experiment. "I ask them to look at the pattern of metabolic changes," she says. "Are the pathways being perturbed in concert, in general, the

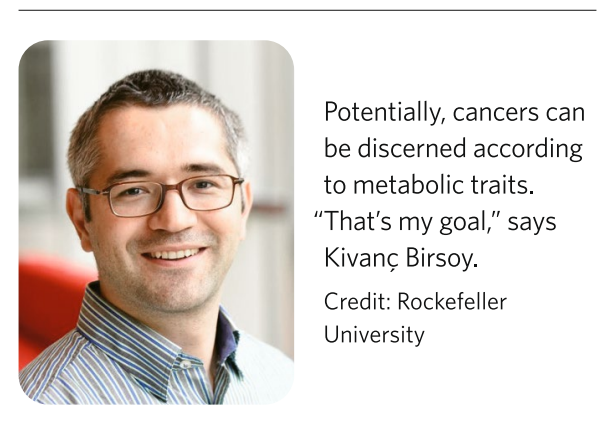

way we saw in the last experiment?" She advises not focusing on a 1.5 -fold increase in one experiment and a 3 -fold change in the next. If the same metabolites are going up or down, she says, that indicates the overall perturbation and a metabolic shift. Experiments done even in slightly different ways can deliver cellular output with a different dynamic range.

Looking at higher levels of TCA intermediates could lead to the hypothesis that the cells are more oxidative, indicating that OXPHOS is more important to the cells' survival, says Finley. "I can test that," she says. Studying metabolism is, in her view, "the starting point to understanding what cells need to do their job," she says. "But you always have to go in and test that." When cells are assessed in different media, answers might differ for each medium because cells solve their "problem" when presented with different parameters. When she and her team measure TCA metabolites, a high level of $\alpha$-keto-glutarate is meaningful only if a specific pathway change has been predicted. "I view measuring metabolites as hypothesis-generating," she says.

"I think you should take whatever tool you can to interrogate metabolism," she says. Her lab is not partial to any one techniquebe it NMR, mass spec or genetics-but rather she integrates results from multiple techniques with cell-biology-based data to see how metabolism is affecting the cell. Labs focused on a certain class of metabolites might have technique preferences because the metabolites are best measured with that one technique. But when taking a global view of how a cell solves a problem, her approach is all about "studying the cell itself." Many variables shape cell metabolism research, such as how long cells have been on a dish and in which media; all can lead to experimental reproducibility issues. In genetics, gene expression is "a static property of sorts" in that a protein will then be made. But in metabolism research, some aspects of reproducibility can be unrealistic, she says. "Metabolism is changing so quickly that you have to look at patterns."

\section{Future metabolism}

Metabolomics remains in need of methods, for example, for labs seeking to connect the metabolic results to genomic or proteomic data as they assess pathways and consider new experiments, says Raftery. Data integration remains challenging, he says. Data itself can be an issue when both 'omics data and the targeted metabolome data are sparse. "Getting enough overlap is sometimes challenging," he says.

Labs might choose do metabolic profiling early in their investigation to determine experimental approaches to connect findings to pathway information and mutational data. They can begin with a live-cell metabolic assessment with Agilent's Analyzer. "I think that's a pretty good first step," says Raftery. After learning about mitochondrial performance in their samples, they can pursue where glucose is going, see which pathways are affected and do metabolic profiling. "That's where we typically come in," he says.

As a tumor grows in vivo, regions within the tumor emerge with different metabolic traits, and a tumor's metabolism changes its microenvironment, too. Researchers need tools to study this heterogeneity related to cancer and stem cells. Hypoxia in a stem cell niche might reinforce a metabolic pathway with important consequences for maintaining stem cell state, says Finley.

His colleagues in cancer genetics increasingly recognize the role cancer metabolism plays, says Birsoy. Metabolism research has not yet been translated to clinical treatments but he hopes that can happen and that metabolism research in general will continue to grow and mature. Studies in cancer cell metabolism will, in his view, yield new insights about cancer biology, metabolic disorders and healthy cellular metabolism, too. As Finley says, "I think we'll be busy for a while."

\section{Vivien Marx}

Technology editor for Nature Methods.

e-mail:v.marx@us.nature.com

Published online: 27 February 2019 https://doi.org/10.1038/s41592-019-0331-0

References

1. Warburg, O., Wind, F. \& Negelein, E. J. Gen. Physiol. 8 519-530 (1927)

2. Vander Heiden, M. G., Cantley, L. C. \& Thompson, C. B. Science 324, 1029-1033 (2009).

3. DeBerardinis, R. J. et al. Proc. Natl Acad. Sci. USA 104, 19345-19350 (2007).

4. Nagana Gowda, G. A. et al. Front. Mol. Biosci. 5, 49 (2018).

5. Nagana Gowda, G. A. et al. Anal. Chem. 88, 4817-4824 (2016).

6. Birsoy, K. et al. Nature 508, 108-112 (2014).

7. Birsoy, K. et al. Nature https://doi.org/10.1038/s41586-019-0945-5 (2019).

8. Intlekofer, A. \& Finley, L. Nat. Metab. https://doi.org/10.1038/ s42255-019-0032-0 (2019). 